\title{
Place in therapy of rituximab in the treatment of granulomatosis with polyangiitis and microscopic polyangiitis
}

This article was published in the following Dove Press journal:

ImmunoTargets and Therapy

7 August 2015

Number of times this article has been viewed

\section{Shivani Shah \\ Duvuru Geetha}

Department of Medicine, Division of Nephrology, Johns Hopkins University School of Medicine, Baltimore, MD, USA
Correspondence: Duvuru Geetha Department of Medicine, Division of Nephrology, Johns Hopkins University School of Medicine, 30I Mason Lord

Drive, Baltimore, MD 21224, USA

$\mathrm{Tel}+\mathrm{I} 4105502820$

Fax + I 4I0 9550485

Email gduvura@jhmi.edu
Abstract: Granulomatosis with polyangiitis and microscopic polyangiitis are small vessel vasculitides characterized by circulating antineutrophil circulating antibodies. Standard treatment for active severe disease has consisted of cyclophosphamide with glucocorticoids with or without plasmapheresis, which achieves approximately $75 \%$ sustained remission, but carries significant adverse effects such as malignancy, infertility, leukopenia, and infections. The role of B cells in the pathogenesis of anti-neutrophil circulating antibodies-associated vasculitis has been established, and as such, rituximab, a monoclonal anti-CD20 antibody, has been studied in treatment of active granulomatosis with polyangiitis and microscopic polyangiitis (induction) and in maintaining remission. Rituximab has been shown to be effective in inducing remission in several retrospective studies in patients with refractory disease or cyclophosphamide intolerance. The RAVE and RITUXVAS trials demonstrated rituximab is a noninferior alternative to standard cyclophosphamide-based therapy; however, its role in elderly patients and patients with severe renal disease warrants further investigation. Rituximab has been compared with azathioprine for maintaining remission in the MAINRITSAN trial and may be more efficacious in maintaining remission in patients treated with cyclophosphamide induction. Rituximab is not without risks and carries a similar adverse event risk rate as cyclophosphamide in randomized control trials. However, its use can be considered over cyclophosphamide in patients who have relapsing or refractory disease or in young patients seeking to preserve fertility.

Keywords: rituximab, ANCA-associated vasculitis, GPA, MPA, induction therapy, maintenance therapy

\section{Introduction}

Granulomatosis with polyangiitis (GPA) and microscopic polyangiitis (MPA) are rare small vessel vasculitides, which often involve the kidneys, upper and lower respiratory tracts, joints, skin, and nervous system. The presence of circulating antineutrophil cytoplasmic antibodies (ANCA) is characteristic of these disease processes and is detected in $74.5 \%$ and $\sim 90 \%$ of patients with MPA and severe GPA, respectively. ${ }^{1-4}$ Typically, GPA is associated with the presence of cytoplasmic ANCA, which is directed toward proteinase-3 (PR3), and MPA is associated with perinuclear ANCA directed toward myeloperoxidase (MPO). ${ }^{1}$ Due to the presence of circulating ANCA, these vasculitides are called ANCA-associated vasculitis (AAV).

Approximately $75 \%-90 \%$ of patients with AAV develop renal involvement during the course of their disease. ${ }^{1}$ Clinically, patients develop a rapidly progressive glomerulonephritis with the evidence of substantial decrease in creatinine clearance, microscopic hematuria, red blood cell casts, and proteinuria. Histologic findings consist 
of necrotizing, crescentic glomerulonephritis on light microscopy with a paucity of immune deposits on immunofluorescence.

Standard of care for induction remission includes cyclophosphamide and glucocorticoid therapy with or without plasmapheresis. ${ }^{5,6}$ More than $75 \%$ of patients achieve sustained disease remission with this regimen. Unfortunately, about half of patients develop relapsing disease, and exposure to cyclophosphamide increases the risk of infections, malignancy, leukopenia, infertility, and bladder toxicity. ${ }^{7}$

B-cell activity has been associated with active or untreated disease in AAV. Popa et al found the percentage of activated $B$ cells by cytometric assessment was higher in patients with active GPA compared to patients in remission and healthy controls. ${ }^{8}$ B-cell activating factor of the TNF family (BAFF), which plays a critical role in B-cell development and survival and may contribute to autoantibody production, has also been implicated to correlate with disease. Krumbholz et al showed BAFF levels were higher in patients with GPA compared to healthy controls, and more so in patients who were not treated with immunosuppression, but did not find a correlation with active disease. ${ }^{9}$ A later study demonstrated BAFF levels in patients with active MPA was two times higher compared to patients in remission and six times higher compared to healthy controls, suggesting that B-cell activity also plays a role in MPA. ${ }^{10}$ Given the central role of B cells in the pathogenesis of AAV as producers of ANCA and in their capacity to act as antigen presenting cells, B-cell depleting therapy was tested as an alternative treatment strategy for remission induction in AAV.

Rituximab is a chimeric monoclonal anti-CD20 antibody that targets B cells. Specifically, it has been shown to induce antibody-dependent cellular cytotoxicity and complementdependent cytotoxicity of B cells. ${ }^{11}$ Consequently, rituximab has been studied in non-Hodgkin's lymphoma, chronic lymphocytic leukemia, and rheumatoid arthritis, and has garnered US Food and Drug Administration (FDA) approval for these indications. $^{12,13}$

\section{Rituximab for induction therapy}

The role of rituximab for induction remission has been illustrated in several reports and has been subsequently validated by the RAVE and RITUXVAS trials as a noninferior alternative to cyclophosphamide and glucocorticoid induction (Table 1). ${ }^{14,15}$ Specks et al reported a case of rituximab use in one patient with relapsing GPA and showed complete remission with $375 \mathrm{mg} / \mathrm{m}^{2}$ dosed weekly for 4 weeks. ${ }^{16}$ The patient was subsequently treated with the same dose of rituximab after another relapse and again achieved complete remission. In another report, ten patients with GPA and one patient with MPA with either refractory disease to treatment with cyclophosphamide or contraindications to cyclophosphamide use were treated with rituximab $375 \mathrm{mg} / \mathrm{m}^{2}$ weekly for 4 weeks, and all achieved remission at 6 months with general tolerability to the regimen. ${ }^{17}$ In a retrospective, multicenter report of 65 patients who were treated with rituximab (either weekly $375 \mathrm{mg} / \mathrm{m}^{2} \times 4$ or biweekly 1,000 mg $\left.\times 2\right), 49(75 \%)$ achieved complete remission and 15 (23\%) obtained partial remission. ${ }^{18}$ In a prospective, open label trial by Keogh et al, ten patients with active anti-PR3 vasculitis and refractory disease or intolerance to cyclophosphamide were treated with rituximab $375 \mathrm{mg} / \mathrm{m}^{2}$ weekly for 4 weeks. ${ }^{19}$ Similar to prior reports, all patients achieved remission, but the follow-up period was 3 months. In this study, patients had all prior immunosuppression discontinued before enrollment, but they were not required to undergo a washout period. Therefore, it is possible that there may have been some contributing effect from prior treatment with cyclophosphamide to remission.

The RITUXVAS trial assessed the efficacy of rituximab with intravenous cyclophosphamide and glucocorticoids compared to intravenous cyclophosphamide and glucocorticoids in the induction of remission in patients with newly diagnosed AAV and renal involvement. ${ }^{15}$ The trial enrolled 44 patients who were randomized by a 3:1 ratio in favor of rituximab to better characterize potential adverse events with rituximab. Patients who required plasmapheresis were treated prior to randomization. All patients received $1 \mathrm{~g}$ of methylprednisolone intravenously followed by $1 \mathrm{mg} / \mathrm{kg} / \mathrm{d}$ of prednisolone tapered to $5 \mathrm{mg} / \mathrm{d}$ by 6 -month follow-up. The rituximab group received rituximab $375 \mathrm{mg} / \mathrm{m}^{2}$ weekly for 4 weeks and intravenous cyclophosphamide $15 \mathrm{mg} / \mathrm{kg}$ with the first and third rituximab infusion. The rational for concurrent cyclophosphamide use was the concern that the therapeutic effect of rituximab would not be immediate, and therefore, cyclophosphamide was given to provide early disease control. This group was not treated with immunosuppression to maintain remission. The control group was treated with intravenous cyclophosphamide $15 \mathrm{mg} / \mathrm{kg}$ for a minimum of 3 months and a maximum of 6 months to receive a total of six to ten doses. After this treatment, they were given azathioprine for remission maintenance. All patients carried a new diagnosis of AAV and had renal involvement. The median baseline estimated glomerular filtration rate (eGFR) was $20 \mathrm{~mL} / \mathrm{min} / \mathrm{m}^{2}$ and $12 \mathrm{~mL} / \mathrm{min} / \mathrm{m}^{2}$ in the rituximab and control groups, respectively. At 12 months, $76 \%(25 / 33)$ in the rituximab group versus $82 \%(9 / 11)$ in 


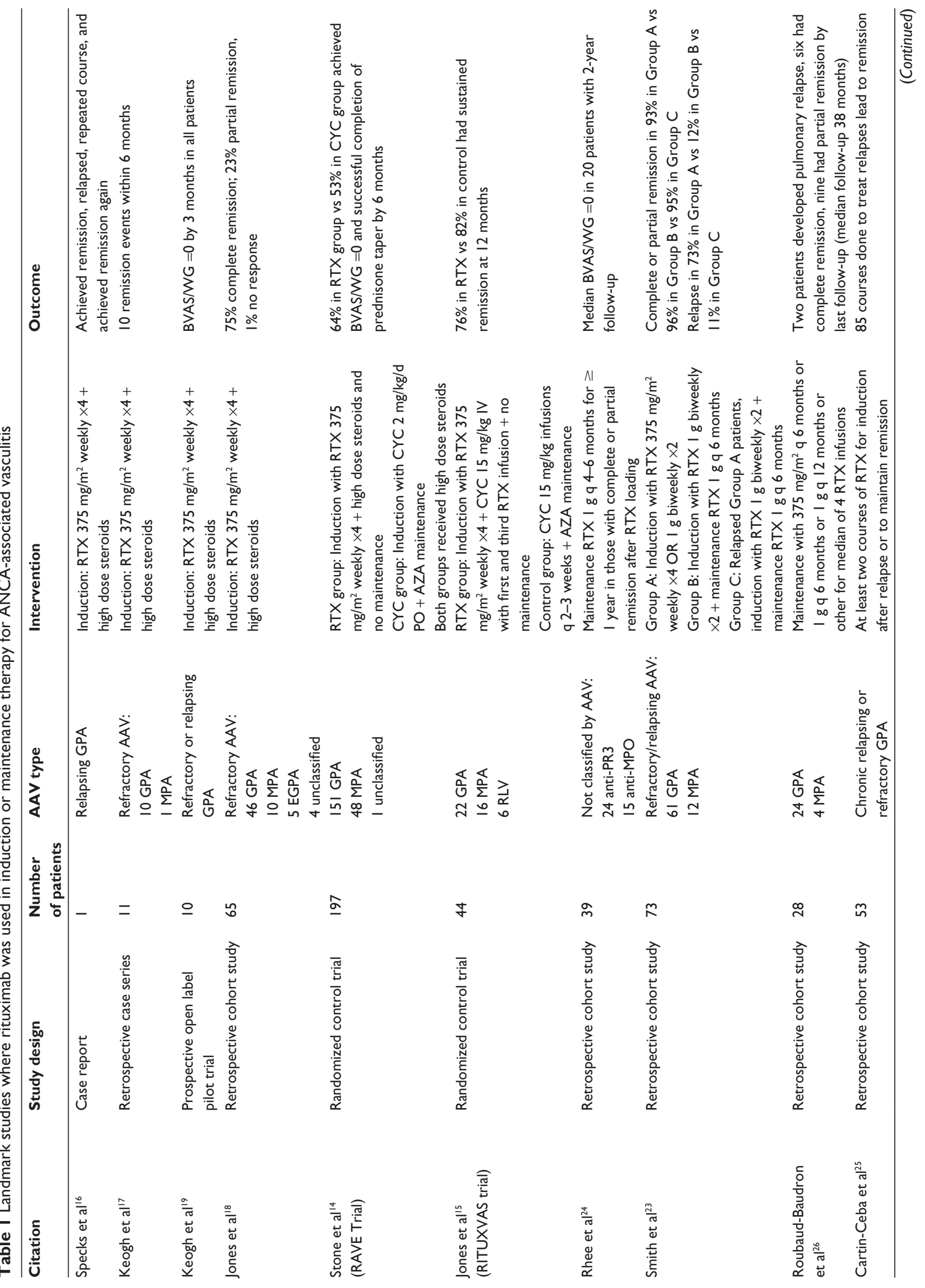


the control group achieved sustained remission. The authors concluded the use of rituximab could decrease the exposure to cyclophosphamide, maintenance of immunosuppression, and their related toxicities although there was no difference in safety observed between the two groups, and both groups were associated with $18 \%$ mortality.

In contrast to the RITUXVAS trial, the RAVE trialtreated patients with either oral cyclophosphamide or rituximab only. ${ }^{14}$ In addition, the follow-up period was 6 months as compared to 12 months in the RITUXVAS study. The RAVE trial was a multicenter, double-blind, double-dummy, noninferiority study where 197 patients with newly diagnosed or relapsing AAV, severe disease manifestations, and Birmingham Vasculitis Score/Wegener's granulomatosis (BVAS/WG) score $\geq 3$ were treated with four weekly infusions of rituximab $375 \mathrm{mg} / \mathrm{m}^{2}$ or cyclophosphamide $2 \mathrm{mg} / \mathrm{kg} / \mathrm{d}$ (renal dose adjusted) for 3-6 months. Patients were excluded if they had a creatinine $>4 \mathrm{mg} / \mathrm{dL}$ or required mechanical ventilation for alveolar hemorrhage. After achieving remission, the patients in the control group were treated with azathioprine for maintenance of remission, whereas the rituximab arm was treated with placebo. The primary outcome was disease remission defined as BVAS/ WG score of 0 without prednisone use at 6 months. In the rituximab group, 64\% (63/99) versus 53\% (52/98) in the control group achieved remission, which fulfilled criteria for noninferiority $(P<0.001)$. Although the baseline mean estimated GFR was lower in the rituximab group than the control group ( $53 \mathrm{~mL} / \mathrm{min}$ vs $69 \mathrm{~mL} / \mathrm{min}, P=0.04)$, there was no significant difference between the proportion of renal patients reaching the primary endpoint in either group. Rituximab yielded higher remission rates in patients with relapsing disease (67\% vs $42 \%$ in control group, $P=0.01$ ). There was no significant difference in adverse events, even though the oral cyclophosphamide regimen is associated with greater toxicity compared to the intravenous regimen that was used in the RITUXVAS study since it provides a higher cumulative dose. ${ }^{20}$ Given its noninferiority to cyclophosphamide, rituximab was approved for induction remission of AAV by the US FDA in April 2011.

Long-term follow-up in both the RAVE and RITUXVAS trials have demonstrated similar outcomes between the rituximab-treated arm and cyclophosphamide/azathioprine-treated arm. ${ }^{21,22}$ At 18-month follow-up in the RAVE trial, 39\% of the rituximab patients compared to $33 \%$ of the control group maintained complete remission $(P=0.32)$, and there was no significant difference in interval improvement in estimated creatinine clearance between groups. ${ }^{21}$ There was also no 
significant difference between groups with regard to rates of total adverse events or serious adverse events. There were two deaths in each group at the end of the follow-up period.

In contrast, the RITUXVAS trial's follow-up period was extended to a total of 24 months. ${ }^{22}$ The primary endpoint was a composite of death, end stage renal disease (ESRD), and relapse. Approximately $42 \%$ (14/33) compared to $36 \%$ (4/11) reached the primary endpoint in the rituximab and control groups, respectively $(P=1.00)$. Relapse rates were similar between groups as well ( $21 \%$ vs $18 \%$ in rituximab vs control groups, respectively, $P=1.00$ ), despite the rituximab group not receiving any maintenance outside of low-dose glucocorticoids following induction. All relapses in the rituximab group occurred after B cell return. The cumulative survival of patients without ESRD or relapse was $58 \%$ in the rituximab group compared to $73 \%$ in the control group, and this did not reach statistical significance $(P=0.37)$. Mortality rates were also similar between the two groups. Overall, rituximab with intravenous cyclophosphamide without maintenance seems to confer similar outcomes as intravenous cyclophosphamide induction with azathioprine maintenance in patients with newly diagnosed AAV and renal involvement.

\section{Rituximab use for maintenance of remission}

Approximately one-half of patients who establish remission develop relapse within the following 5 years, and about $10 \%$ of patients have refractory disease. ${ }^{23}$ Consequently, rituximab was used in patients with refractory and relapsing disease with standard therapy and has demonstrated efficacy in maintaining remission and preventing future relapses in several retrospective studies (Table 1). Rhee et al evaluated the effect of rituximab in 39 patients with partial or complete remission and found that all maintained remission by 1 year and, therefore, had a reduced need for other immunosuppressive agents. ${ }^{24}$ In another retrospective study, 53 patients with chronic relapsing or refractory GPA were treated with a median of four doses of rituximab, and all achieved remission in relapsing disease, and remission was maintained in all patients who were treated with rituximab prior to B-cell reconstitution. ${ }^{25}$ This study suggested that $\mathrm{B}$-cell reconstitution is a harbinger of relapse, and therefore, prolonged B-cell depletion may avert relapse. Roubaud-Baudron et al examined 28 patients with MPA or GPA, most of whom had relapsing disease, who received two or more maintenance infusions of rituximab between 2003 and $2010 .{ }^{26}$ Twenty-six patients had complete or partial remission at last follow-up, where minimum follow-up time was 1 year. Rituximab was also shown to be efficacious in a retrospective study where patients received fixed interval dosing of $1 \mathrm{~g}$ infusions every 6 months over 2 years. ${ }^{23}$ With this protocol, patients experienced a reduction in relapse rates and prolonged disease remission.

Pendergraft et al retrospectively evaluated long-term maintenance immunosuppression with rituximab. ${ }^{27}$ One hundred seventy-two patients in remission or converting from another maintenance therapy, of whom 57\% were anti-MPO positive, underwent continuous B-cell depletion with scheduled rituximab infusions of 1,000 mg every 4 months for up to 7 years. The median follow-up time was 2.1 years. Approximately $5 \%$ of patients developed major relapse, defined as BVAS/WG score $\geq 3$. About $20 \%$ of patients experienced minor relapse, characterized as BVAS/WG $=2$. The authors concluded that the fixed schedule of rituximab was safe and effective, although indefinite scheduling did not seem practical and further studies would be needed to assess the duration of rituximab therapy for remission maintenance.

Current evidence supports the use of azathioprine or methotrexate (in patients with no or mild renal disease) as maintenance immunosuppression, although there was a trend toward more serious adverse events with methotrexate when compared to azathioprine in the WEGENT trial. ${ }^{28,29}$ Given that retrospective data suggest maintenance therapy with successive rituximab infusions may be effective in preventing relapse, this approach had not been prospectively compared with azathioprine until the MAINRITSAN trial. ${ }^{30}$ This study enrolled 115 patients with newly diagnosed or relapsing MPA, GPA or renal-limited AAV who were in complete remission after induction with glucocorticoids and cyclophosphamide therapy. Patients were excluded if they received rituximab or another biologic treatment prior to enrollment. Within 1 month of established remission and last cyclophosphamide pulse, patients were randomly assigned to receive rituximab or azathioprine maintenance therapy. Patients randomized to the rituximab arm were treated with $500 \mathrm{mg}$ infusions on days 0 and 14, and then at months 6,12 , and 18 . The control group received a tapered azathioprine regimen with $2 \mathrm{mg} / \mathrm{kg} / \mathrm{d}$ for the first 12 months, followed by $1.5 \mathrm{mg} / \mathrm{kg} / \mathrm{d}$ for 6 months, and $1 \mathrm{mg} / \mathrm{kg} / \mathrm{d}$ for 4 months. Patients were also on a prednisone taper in both groups and maintained on prednisone $5 \mathrm{mg} / \mathrm{d}$ for at least 18 months after randomization. The primary endpoint was the percentage of patients with reappearance or worsening of disease with BVAS score $>0$ and involvement of one or more major organ, a life-threatening manifestation, or both at the end of the follow-up period of 28 months. 
Seventeen patients $(29 \%)$ in the azathioprine compared to three patients $(5 \%)$ in the rituximab group $(P=0.002)$ developed major relapse at the end of the follow-up period. The number needed to treat to prevent one major relapse was four patients ( $95 \%$ CI: 3-9), and it seems relapse rates were low in the rituximab group while they were receiving infusions. In this study, the patients who developed relapse in the rituximab group did not have B-cell reconstitution, suggesting that the absence B-cell reconstitution does not necessarily correlate to the lack of relapse. ${ }^{31}$

There were several limitations of the MAINRITSAN trial. The study population consisted mostly of patients with GPA, and the trial was nonblinded. The number of relapses was fewer than anticipated in both groups, and this was attributed to continued glucocorticoid use through 18 months of follow-up. Additionally, the follow-up period was criticized as too short to determine whether more relapses would have occurred after cessation of rituximab infusions. ${ }^{32}$ The study was also criticized for using an azathioprine taper, and it may have skewed the results in favor of rituximab. ${ }^{33}$ Furthermore, the long-term safety profile of rituximab in this population is not known unlike that of azathioprine. ${ }^{33}$ As far as severe adverse events by the end of the follow-up period, both groups had similar rates of infection and malignancy, but it is unclear whether the malignancy risk is attributable to the maintenance regimens or cyclophosphamide-induction therapy.

However, it is still unclear what maintenance regimen should be used following induction with rituximab. In the RAVE trial, all immunosuppressions were discontinued in the rituximab arm after 6 months, and in the RITUXVAS trial, low-dose glucocorticoids were employed 6 months after rituximab induction. ${ }^{14,15}$ However, approximately one-half of the patients develop relapse within 2 years of rituximab induction. The RITAZAREM trial (NCT01697267) is currently enrolling patients to further investigate the role of rituximab versus azathioprine as maintenance immunosuppression in patients with relapsing AAV who underwent induction therapy with rituximab. Approximately 190 participants from Europe, North America, and Australia are being sought to be randomized to $1,000 \mathrm{mg}$ rituximab infusion every 4 months for 2 years or azathioprine. After the follow-up period of 4 years, the primary endpoint is the time to disease relapse.

\section{Rituximab induction in severe renal disease}

Rituximab induction in patients with severe renal disease is limited. RITUXVAS included $9 / 44$ patients requiring dialysis at enrollment, and more than one-third of the total cohort had an estimated GFR $<15 \mathrm{~mL} / \mathrm{min} / 1.73 \mathrm{~m}^{2}{ }^{22}$ Approximately $53 \%$ of patients in the rituximab arm survived and achieved an estimated GFR $>15 \mathrm{~mL} / \mathrm{min} / 1.73 \mathrm{~m}^{2}$. The RAVE trial excluded patients with creatinine $>4 \mathrm{mg} / \mathrm{dL}$, but $20 \%$ and $16 \%$ of patients in the rituximab and cyclophosphamide-azathioprine groups, respectively, had creatinine clearances $<30 \mathrm{~mL} /$ min. ${ }^{21}$ Patients with renal involvement had similar rates of remission in both groups.

A single multicenter retrospective study currently under review suggests that rituximab induction in MPA and GPA patients with newly diagnosed or relapsing disease and estimated GFR $<20 \mathrm{~mL} / \mathrm{min} / \mathrm{m}^{2}$ is effective and relatively safe after 6-month follow-up. Fourteen patients were included from five centers from the US and Europe. Six had GPA and two had relapsing disease. Two patients had ANCA negative serologies. The median estimated GFR at presentation was $12.5 \mathrm{~mL} / \mathrm{min} / 1.73 \mathrm{~m}^{2}$, and half of the cohort required dialysis at onset. Five were treated with plasmapheresis. Patients were treated with one course of rituximab (two 1,000 mg biweekly infusions or four $375 \mathrm{mg} / \mathrm{m}^{2}$ weekly infusions). Patients were excluded if they received cyclophosphamide or had the presence of anti-GBM antibodies. By 6-month followup, all patients achieved remission, which was defined as stabilization or improvement in serum creatinine, resolution of hematuria, and absence of extra-renal signs of vasculitis for 1 month. Five of seven patients were able to discontinue dialysis by 6 months. The median eGFR at 6 months in the 11 patients who were not ESRD and completed 6-month follow-up was $31 \mathrm{~mL} / \mathrm{min} / 1.73 \mathrm{~m}^{2}$. All patients were treated with glucocorticoids during their induction and were weaned to prednisone $10 \mathrm{mg} / \mathrm{d}$ or less by 6 months. Only six patients had serum B cells quantified, but all six experienced depletion. Three patients developed severe infections requiring hospitalization within the 1st year following remission induction. Although this study has limitations such as retrospective design, small cohort size, and short follow-up, it does show some promise for the efficacy of rituximab in this population.

\section{Rituximab in the elderly}

Elderly patients with AAV, often present with MPA, have more severe renal dysfunction at presentation and higher rates of infection. ${ }^{34}$ They have a higher mortality risk due to both their disease and adverse effects related to induction therapy with cyclophosphamide. ${ }^{34}$ Data surrounding the efficacy and safety of rituximab in this population with AAV are sparse. One single center, retrospective study involving 31 patients with mean age of 71 years at the time of rituximab 
induction therapy found that $97 \%$ (30/31) of patients were able to achieve remission with mean time to remission of 57 days. ${ }^{35}$ One-year survival in patients with at least 1-year follow-up was $100 \%$. Although $71 \%$ of patients had glomerulonephritis at baseline, only one patient progressed to ESRD during the follow-up period. There were five episodes of serious infections in this group, highlighting the greater risk of infection in this group even with rituximab use.

\section{Rituximab use in recurrence postrenal transplant}

Renal transplant is a safe and effective treatment for patients who develop ESRD due to AAV. ${ }^{36}$ Recurrent AAV in the allograft is uncommon in this era of potent immunosuppressive drug choices, but rates of $9 \%-40 \%$ have been reported. ${ }^{36-38}$ Experience using rituximab for the treatment of recurrent disease in a transplanted kidney is limited to case reports. Three reports describing eight patients with recurrence of AAV postrenal transplant demonstrated that $7 / 8$ patients were able to achieve remission with rituximab and glucocorticoid induction therapy. ${ }^{38-40} \mathrm{~A}$ single patient in the cohort described by Murakami et al experienced recurrence that was refractory to treatment with rituximab and cyclophosphamide. ${ }^{39}$

\section{Rituximab during pregnancy}

Data surrounding the safety of rituximab in pregnancy is limited. In a study by Pendergraft et al evaluating the efficacy of rituximab as maintenance immunosuppression, patients were counseled regarding contraception, and urine human chorionic gonadotropin (HCG) was tested prior to rituximab administration, but five women achieved six pregnancies during their treatment. ${ }^{27}$ There were five healthy deliveries and one fetal demise at 15 weeks. In another report by Pendergraft et al, which may have included some of the aforementioned patients, the author reported eight pregnancies between six women treated with rituximab. ${ }^{41}$ Most patients were thought to have conceived soon after infusion. One patient in this cohort suffered a second trimester miscarriage due to BeckwithWiedemann syndrome, which was considered unrelated to rituximab exposure. Another patient developed worsening of reactive airway disease, but the remaining pregnancies were uneventful. The findings in this report suggest that perhaps first trimester exposure may be safe, but further studies are warranted.

Rituximab crosses the placenta in the second and third trimester such that fetal serum levels are similar or higher than maternal serum levels. ${ }^{42} \mathrm{~A}$ case report where rituximab was used in treatment of idiopathic thrombocytopenia in the third trimester illustrated that rituximab was present at higher levels in the neonate and had resulted in undetectable B-cell counts. ${ }^{43}$ However, the baby had no congenital defects, after 6 months B-cell levels were normalized, and vaccination titers after 10 months were adequate. ${ }^{43}$ In a global safety database by Chakravarty et al, the outcomes of 153 pregnancies with maternal exposure to rituximab were described. ${ }^{44}$ There were 90 (59\%) live births of which 68 (76\%) were full term, 22 were premature (defined as $<37$ weeks), and no live births occurred before 30 -weeks gestation. One neonate died at 6 weeks. There were $33(22 \%)$ spontaneous abortions and $28(18 \%)$ terminated pregnancies. There were 21 patients who received rituximab after established pregnancy, none of the offspring incurred congenital malformations, and there were no neonatal deaths resulting from these pregnancies. Unfortunately, the maternal exposure to rituximab was confounded by maternal treatment with other teratogenic medications and severe underlying diseases such as lymphoma and autoimmune cytopenias and other autoimmune diseases.

Given these findings, Ostensen et al and Chakravarty et al recommend avoiding pregnancy for 6-12 months following exposure to rituximab. ${ }^{42,44}$ Rituximab is classified as Category $\mathrm{C}$, where risk to the fetus cannot be ruled out since there are no controlled studies in women. ${ }^{45}$ Risks and benefits of administration must be considered. Of note, excretion in breast milk is unknown.

\section{Rituximab and timing of plasma exchange}

Plasma exchange has been shown to be beneficial in patients with AAV and severe renal disease (creatinine $>5.8 \mathrm{mg}$ / dL) by reducing renal morbidity and short-term mortality. ${ }^{5}$ Plasma exchange is also indicated in severe lung involvement and alveolar hemorrhage. Thus, patients may be treated with both rituximab and plasma exchange in severe cases. There are no available guidelines that dictate the optimal timing of rituximab infusion relative to plasma exchange. Some authors recommend waiting to dose rituximab until after completion of all plasma exchange treatments. ${ }^{45,46}$ Alternative strategies include timing rituximab infusion 48-72 hours prior to the first plasma exchange or holding plasma exchange the day following rituximab infusion. ${ }^{46,47}$ A study of the influence of plasma exchange on the pharmacokinetics of rituximab (NCT00820469) has been completed, but the results are currently unavailable. The primary outcome is to compare plasma concentrations of rituximab in patients treated with rituximab alone versus rituximab and plasma exchange, and 
one of the secondary outcomes is to determine the amount of rituximab removed with each exchange. The results of this study will likely shape our practice in this area.

\section{Rituximab-dosing regimen}

A few dosing regimens have been reported for the induction of remission in AAV. Most studies including the RAVE and RITUXVAS trials used a regimen based on what is used to treat lymphoma: four weekly infusions of $375 \mathrm{mg} / \mathrm{m}^{2}{ }^{14,15,48}$ Other studies have used dosing that was approved for rheumatoid arthritis, two 1,000 mg infusions given 2 weeks apart. ${ }^{18,49,50}$ One single center, retrospective study assessed the efficacy of single dose $375 \mathrm{mg} / \mathrm{m}^{2}$ dosing on achieving remission and time to relapse and found a probability of $80 \%$ complete remission by 3 months and a median of 27 months to relapse. ${ }^{51}$ The study by Jones et al used both $375 \mathrm{mg} / \mathrm{m}^{2}$ $\times 4$ and $1,000 \mathrm{mg} \times 2$ regimens for induction and did not find a significant difference in remission rates based on the two regimens. ${ }^{18}$

There are no prospective trials comparing any rituximab induction regimens. A panel of experts from the United Kingdom developed recommendations for rituximab use in AAV. Based on a literature review at that time, they concluded that either four weekly infusions of $375 \mathrm{mg} / \mathrm{m}^{2}$ or two biweekly infusions of $1,000 \mathrm{mg}$ were acceptable options as induction regimens. ${ }^{48}$ The two dose courses provide a lower cumulative dose of rituximab and less drug wastage.

There are no guidelines for optimal rituximab dosing for the maintenance of immunosuppression. The MAINRITSAN trial used $500 \mathrm{mg}$ rituximab infusions given close to the time of remission and then every 6 months for 18 months, whereas other studies used 1,000 mg infusions every 4-6 months,

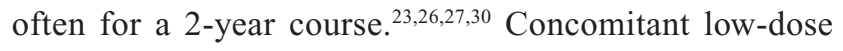
glucocorticoids are not required.

\section{Rituximab, infections, hypogammaglobulinemia, and infection prophylaxis recommendations}

Rituximab is a potent immunosuppressant, and therefore increases the risk of infections. Rates of serious infections in both the RAVE and RITUXVAS trials demonstrate levels comparable to cyclophosphamide treatment. ${ }^{15,21}$ However, there was a higher incidence specifically of pneumonia in the cyclophosphamide-azathioprine arm of the RAVE trial at 18-month follow-up ( $P=0.03)$. In the RITUXVAS trial, both arms had approximately $18 \%$ rates of serious infections at 12-month follow-up. It is difficult to distinguish whether the risk of infection seen in patients treated with rituximab is due solely to rituximab exposure, prior immunosuppression, or concomitant immunosuppressant agents, but it is probably a combination of all of these factors.

Rituximab also carries a boxed warning for reactivation of hepatitis B, resulting in fulminant hepatitis, liver failure, and death based on several reports. ${ }^{52-55}$ Given this risk, all patients are recommended to undergo serologic testing for hepatitis B serologies prior to rituximab dosing. ${ }^{46}$ If treatment is unavoidable, patients with active hepatitis B and HBsAg positivity are recommended lamivudine prophylaxis. ${ }^{54,56}$ There is some evidence to suggest entecavir prophylaxis in patients with resolved hepatitis B infections may also reduce reactivation. ${ }^{57}$ A randomized control trial in People's Republic of China assessed the efficacy of entecavir compared to lamuvidine in preventing reactivation of hepatitis $B$ in patients with lymphoma and surface antigen positivity treated with rituximab as a part of therapy. ${ }^{58}$ They found that in this population, entecavir leads to lower rates of reactivation than lamuvidine. Although a similar study in AAV is lacking, one could consider entecavir prophylaxis over lamuvidine.

Progressive multifocal leukoencephalopathy due to the polyoma virus, JC virus, has been associated with rituximab use in oncologic, transplant, and systemic lupus erythematosus patients. ${ }^{38,48}$ There has also been one report of progressive multifocal leukoencephalopathy in a patient with GPA and breast cancer who was treated with rituximab and cyclophosphamide. ${ }^{45}$ The virus is present in a latent form in approximately $80 \%$ of the population. It is unclear whether it is exposure to rituximab, concomitant immunosuppression, or a generally immunocompromised state that leads to exacerbation or reactivation of the JC virus; however, there is an FDA warning about this risk associated with rituximab use.

Rituximab induced hypogammaglobulinemia is common, but severe forms occur in $<5 \%$ of treated patients. ${ }^{45,48}$ In one series of lymphoma patients treated with rituximab, 38.54\% of patients with normal serum IgG levels prior to rituximab exposure developed hypogammaglobulinemia after treatment with rituximab. ${ }^{59}$ Patients who received maintenance rituximab seemed to be at higher risk. In the 18-month follow-up of the RAVE trial, serum levels of IgG did not differ significantly between the cyclophosphamide and rituximab groups at 18 months (mean [standard deviations] IgG levels were 766[265] and 808[363] in the rituximab and cyclophosphamide treated arms, respectively $[P=0.50]) .{ }^{21}$ However, serum IgG levels decreased from baseline in both groups after treatment. Despite this, they reported no significant association between hypogammaglobulinemia and severe infection risk. 
Some recommend replacement with intravenous immune globulin (IVIG) in serious infections. ${ }^{56}$

There is a tangible risk of pneumocystis jirovecii pneumonia (PJP) in patients treated with rituximab, since B cells have been found to play an important role in fighting this infection; and therefore, prophylactic antibiotic treatment is recommended while patients receive rituximab. ${ }^{60,61} \mathrm{In}$ the non-HIV population, prophylaxis is suggested when the risk of PJP exceeds 3.5\% ${ }^{62}$ The incidence of PJP has not been quantified in patients receiving rituximab; however, a retrospective review of records of patients who received rituximab at the Mayo Clinic between 1998 and 2011 discovered 30 patients were diagnosed with PJP. ${ }^{61}$ Most patients in this cohort were being treated for hematologic malignancies, but one patient had GPA. Three out of the 30 patients received rituximab as their sole immunosuppressive therapy. Twenty nine patients did not receive PJP prophylaxis. Overall mortality in this cohort was 30\%. Based on these findings, PJP prophylaxis is recommended. The optimal duration of prophylaxis is unclear, but some authors recommend continuing prophylaxis until B-cell reconstitution. ${ }^{46}$ However, the authors of the MAINRITSAN study monitored CD4 counts and discontinued prophylaxis when CD4 $>250$ cells $/$ cc. $^{30}$

Patients who receive rituximab are also recommended to undergo vaccinations 1 month prior to infusions. ${ }^{48}$ Although this may be difficult to schedule in the patient with newly diagnosed, aggressive disease, it should be considered in patients who have not been vaccinated and are being considered for maintenance immunotherapy.

\section{Conclusion}

Rituximab is a comparable alternative to cyclophosphamide with regard to efficacy and safety for the induction of remission

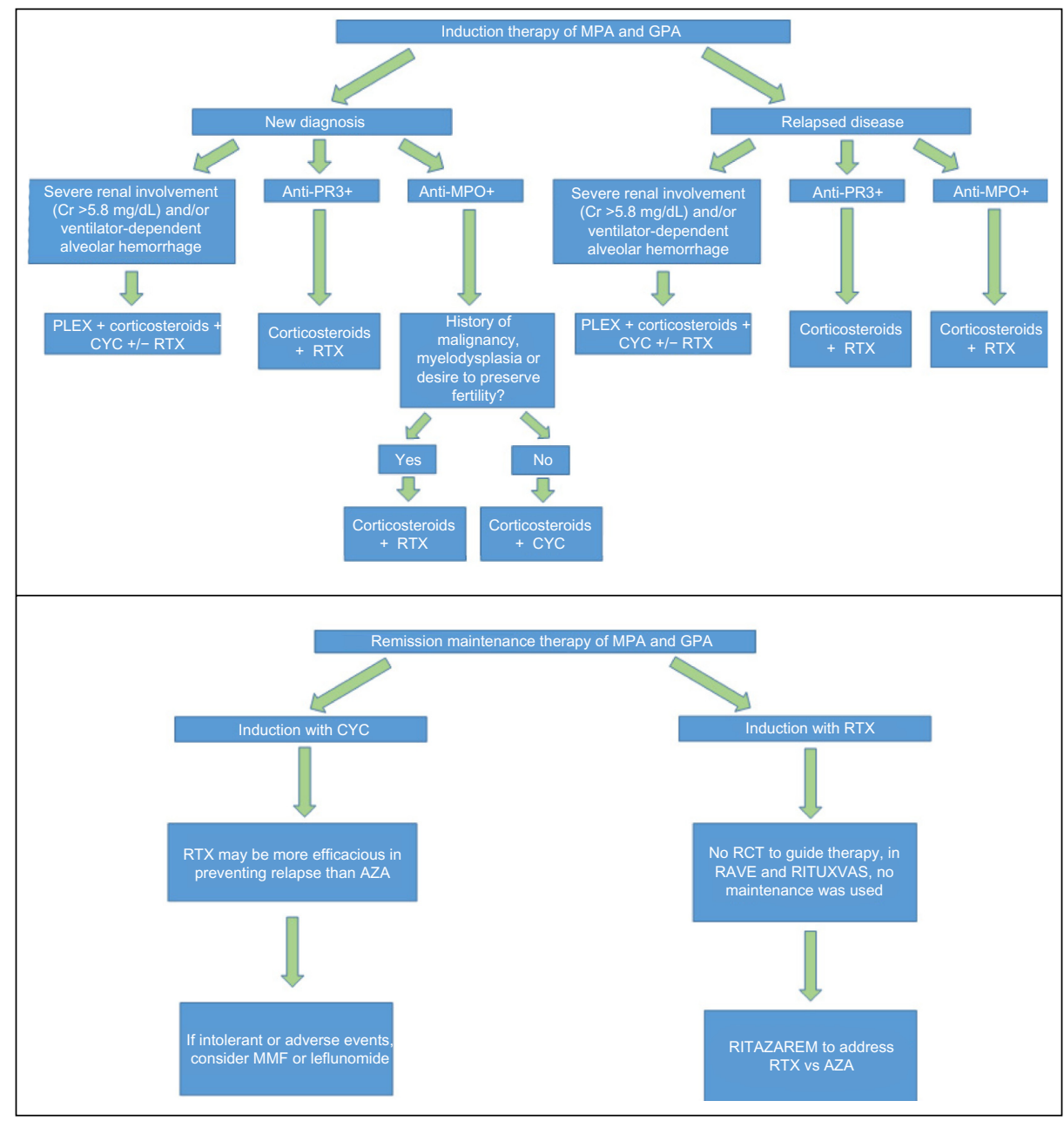

Figure I Treatment algorithm for AAV.

Abbreviations: AAV, antineutrophil cytoplasmic antibodies-associated vasculitis; MPA, microscopic polyangitis; GPA, granulomatosis with polyangiitis ; PR3, proteinase-3; MPO, myeloperoxidase; CYC, cyclophosphamide; RTX, rituximab; AZA, azathioprine; PLEX, plasma exchange; RCT, randomized controlled trial, Cr, serum creatinine; MMF, mycophenolate mofetil. 
of AAV. Rituximab should be considered in patients with disease refractory to cyclophosphamide therapy and in relapsing disease, particularly those with anti-PR3 positivity and in young patients who wish to preserve fertility (Figure 1). The role of rituximab for remission induction in severely ill AAV patients such as those with severe renal failure requiring dialysis and those with alveolar hemorrhage requiring mechanical ventilation merits further study. Given most studies enrolled patients primarily with GPA, the use of rituximab in patients with MPA, renal limited disease, and ANCA negative pauciimmune glomerulonephritis requires further investigation.

Rituximab has been shown to be an effective alternative to azathioprine for remission maintenance following induction with cyclophosphamide. The role of rituximab in maintenance after induction with rituximab will be elucidated by the RITAZAREM trial.

Further studies are needed to clarify the optimal rituximab dosing strategies for induction and maintenance of remission and duration of rituximab use for maintenance of remission, as well as timing and dosing with concomitant plasmapheresis.

\section{Disclosure}

Duvuru Geetha has served as a consultant to Genentech. The authors report no other conflicts of interest in this work.

\section{References}

1. Franssen CF, Stegeman CA, Kallenberg CG, et al. Antiproteinase 3 - and antimyeloperoxidase-associated vasculitis. Kidney Int. 2000;57(6):2195-2206.

2. Comarmond C, Cacoub P. Granulomatosis with polyangiitis (Wegener): clinical aspects and treatment. Autoimmun Rev. 2014;13(11): 1121-1125.

3. Finkielman JD, Lee AS, Hummel AM, et al; WGET Research Group. ANCA are detectable in nearly all patients with active severe Wegener's granulomatosis. Am J Med. 2007;120(7):.e9-.e643.

4. Guillevin L, Durand-Gasselin B, Cevallos R, et al. Microscopic polyangiitis: clinical and laboratory findings in eighty-five patients. Arthritis Rheum. 1999;42(3):421-430.

5. Jayne DR, Gaskin G, Rasmussen N, et al; European Vasculitis Study Group. Randomized trial of plasma exchange or high-dosage methylprednisolone as adjunctive therapy for severe renal vasculitis. J Am Soc Nephrol. 2007;18(7):2180-2188.

6. Fauci AS, Haynes BF, Katz P, Wolff SM. Wegener's granulomatosis: prospective clinical and therapeutic experience with 85 patients for 21 years. Ann Intern Med. 1983;98(1):76-85.

7. Seo P, Min YI, Holbrook JT, et al; WGET Research Group. Damage caused by Wegener's granulomatosis and its treatment: prospective data from the Wegener's granulomatosis etanercept trial (WGET). Arthritis Rheum. 2005;52(7):2168-2178.

8. Popa ER, Stegeman CA, Bos NA, Kallenberg CG, Tervaert JW. Differential B- and T-cell activation in Wegener's granulomatosis. J Allergy Clin Immunol. 1999;103(5 pt 1):885-894.

9. Krumbholz M, Specks U, Wick M, Kalled SL, Jenne D, Meinl E. BAFF is elevated in serum of patients with Wegener's granulomatosis. JAutoimmun. 2005;25(4):298-302.
10. Xin G, Chen M, Su Y, Xu LX, Zhao MH, Li KS. Serum B-cell activating factor in myeloperoxiase-antineutrophil cytoplasmic antibodiesassociated vasculitis. Am J Med Sci. 2014;348(1):25-29.

11. Johnson P, Glennie M. The mechanisms of action of rituximab in the elimination of tumor cells. Semin Oncol. 2003;30(1 Suppl 2):3-8.

12. Johnson PW, Glennie MJ. Rituximab: mechanisms and applications. Br J Cancer. 2001;85(11):1619-1623.

13. Rath E, Zwerina J, Oppl B, Nell-Duxneuner V. Efficacy and safety of rituximab in rheumatic diseases. Wien Med Wochenschr. 2015; 165(1-2):28-35.

14. Stone JH, Merkel PA, Spiera R, et al; RAVE-ITN Research Group. Rituximab versus cyclophosphamide for ANCA-associated vasculitis. N Engl J Med. 2010;363(3):221-232.

15. Jones RB, Tervaert JW, Hauser T, et al; European Vasculitis Study Group. Rituximab versus cyclophosphamide in ANCA-associated renal vasculitis. $N$ Engl J Med. 2010;363(3):211-220.

16. Specks U, Fervenza FC, McDonald TJ, Hogan MC. Response of Wegener's granulomatosis to anti-CD20 chimeric monoclonal antibody therapy. Arthritis Rheum. 2001;44(12):2836-2840.

17. Keogh KA, Wylam ME, Stone JH, Specks U. Induction of remission by B lymphocyte depletion in eleven patients with refractory antineutrophil cytoplasmic antibody-associated vasculitis. Arthritis Rheum. 2005;52(1):262-268.

18. Jones RB, Ferraro AJ, Chaudhry AN, et al. A multicenter survey of rituximab therapy for refractory antineutrophil cytoplasmic antibodyassociated vasculitis. Arthritis Rheum. 2009;60(7):2156-2168.

19. Keogh KA, Ytterberg SR, Fervenza FC, Carlson KA, Schroeder DR, Specks U. Rituximab for refractory Wegener's granulomatosis: report of a prospective, open-label pilot trial. Am J Respir Crit Care Med. 2006;173(2):180-187.

20. Falk RJ, Jennette JC. Rituximab in ANCA-associated disease. $N$ Engl J Med. 2010;363(3):285-286.

21. Specks U, Merkel PA, Seo P, et al; RAVE-ITN Research Group. Efficacy of remission-induction regimens for ANCA-associated vasculitis. $N$ Engl J Med. 2013;369(5):417-427.

22. Jones RB, Furuta S, Tervaert JW, et al; European Vasculitis Society (EUVAS). Rituximab versus cyclophosphamide in ANCA-associated renal vasculitis: 2-year results of a randomised trial. Ann Rheum Dis. 2015;74(6):1178-1182.

23. Smith RM, Jones RB, Guerry MJ, et al. Rituximab for remission maintenance in relapsing antineutrophil cytoplasmic antibody-associated vasculitis. Arthritis Rheum. 2012;64(11):3760-3769.

24. Rhee EP, Laliberte KA, Niles JL. Rituximab as maintenance therapy for anti-neutrophil cytoplasmic antibody-associated vasculitis. Clin J Am Soc Nephrol. 2010;5(8):1394-1400.

25. Cartin-Ceba R, Golbin JM, Keogh KA, et al. Rituximab for remission induction and maintenance in refractory granulomatosis with polyangiitis (Wegener's): ten-year experience at a single center. Arthritis Rheum. 2012;64(11):3770-3778.

26. Roubaud-Baudron C, Pagnoux C, Méaux-Ruault N, et al; French Vasculitis Study Group. Rituximab maintenance therapy for granulomatosis with polyangiitis and microscopic polyangiitis. J Rheumatol. 2012; 39(1):125-130.

27. Pendergraft WF 3rd, Cortazar FB, Wenger J, et al. Long-term maintenance therapy using rituximab-induced continuous B-cell depletion in patients with ANCA vasculitis. Clin J Am Soc Nephrol. 2014; 9(4):736-744.

28. Jayne D, Rasmussen N, Andrassy K, et al; European Vasculitis Study Group. A randomized trial of maintenance therapy for vasculitis associated with antineutrophil cytoplasmic autoantibodies. $N$ Engl J Med. 2003;349(1):36-44.

29. Pagnoux C, Mahr A, Hamidou MA, et al; French Vasculitis Study Group. Azathioprine or methotrexate maintenance for ANCA-associated vasculitis. N Engl J Med. 2008;359(26):2790-2803.

30. Guillevin L, Pagnoux C, Karras A, et al; French Vasculitis Study Group. Rituximab versus azathioprine for maintenance in ANCA-associated vasculitis. $N$ Engl J Med. 2014;371(19):1771-1780. 
31. Tanna A, Pusey C. Clinical trials. rituximab for maintenance of remission in AAV. Nat Rev Nephrol. 2015;11(3):131-132.

32. Jayne D. Extending the indications for rituximab in ANCA-associated vasculitis. $N$ Engl J Med. 2014;371(19):1839-1840.

33. Lee HA, Alvarado A, Rovin B, et al. Rituximab or azathioprine maintenance in ANCA-associated vasculitis. $N$ Engl $\mathrm{J}$ Med. 2015;372(4):385-387.

34. Harper L, Savage CO. ANCA-associated renal vasculitis at the end of the twentieth century - a disease of older patients. Rheumatology (Oxford). 2005;44(4):495-501.

35. Timlin H, Lee SM, Manno RL, Seo P, Geetha D. Rituximab for remission induction in elderly patients with ANCA-associated vasculitis. Semin Arthritis Rheum. Epub 2015 Feb 20.

36. Geetha D, Eirin A, True K, et al. Renal transplantation in antineutrophil cytoplasmic antibody-associated vasculitis: a multicenter experience. Transplantation. 2011;91(12):1370-1375.

37. Gera M, Griffin MD, Specks U, Leung N, Stegall MD, Fervenza FC. Recurrence of ANCA-associated vasculitis following renal transplantation in the modern era of immunosupression. Kidney Int. 2007; 71(12):1296-1301.

38. Geetha D, Seo P, Specks U, Fervenza FC. Successful induction of remission with rituximab for relapse of ANCA-associated vasculitis post-kidney transplant: report of two cases. Am J Transplant. 2007; 7(12):2821-2825.

39. Murakami C, Manoharan P, Carter-Monroe N, Geetha D. Rituximab for remission induction in recurrent ANCA-associated glomerulonephritis postkidney transplant. Transpl Int. 2013;26(12):1225-1231.

40. Hermle T, Goestemeyer AK, Sweny P, Burns A. Successful therapeutic use of rituximab in refractory Wegener's granulomatosis after renal transplantation. Clin Nephrol. 2007;68(5):322-326.

41. Pendergraft WF 3rd, McGrath MM, Murphy AP, et al. Fetal outcomes after rituximab exposure in women with autoimmune vasculitis. Ann Rheum Dis. 2013;72(12):2051-2053.

42. Ostensen M, Forger F. Treatment with biologics of pregnant patients with rheumatic diseases. Curr Opin Rheumatol. 2011;23(3):293-298.

43. Klink DT, van Elburg RM, Schreurs MW, van Well GT. Rituximab administration in third trimester of pregnancy suppresses neonatal B-cell development. Clin Dev Immunol. 2008;2008:271363.

44. Chakravarty EF, Murray ER, Kelman A, Farmer P. Pregnancy outcomes after maternal exposure to rituximab. Blood. 2011;117(5) 1499-1506.

45. Geetha D, Kallenberg C, Stone JH, et al. Current therapy of granulomatosis with polyangiitis and microscopic polyangiitis: the role of rituximab. J Nephrol. 2015;28(1):17-27.

46. Clain JM, Cartin-Ceba R, Fervenza FC, Specks U. Experience with rituximab in the treatment of antineutrophil cytoplasmic antibody associated vasculitis. Ther Adv Musculoskelet Dis. 2014;6(2):58-74.

47. Jasti S, Coyle T, Gentile T, Rosales L, Poiesz B. Rituximab as an adjunct to plasma exchange in TTP: a report of 12 cases and review of literature. J Clin Apher. 2008;23(5):151-156.

48. Guerry MJ, Brogan P, Bruce IN, et al. Recommendations for the use of rituximab in anti-neutrophil cytoplasm antibody-associated vasculitis. Rheumatology (Oxford). 2012;51(4):634-643
49. Taylor SR, Salama AD, Joshi L, Pusey CD, Lightman SL. Rituximab is effective in the treatment of refractory ophthalmic Wegener's granulomatosis. Arthritis Rheum. 2009;60(5):1540-1547.

50. Mansfield N, Hamour S, Habib AM, et al. Prolonged disease-free remission following rituximab and low-dose cyclophosphamide therapy for renal ANCA-associated vasculitis. Nephrol Dial Transplant. 2011;26(10):3280-3286

51. Turner-Stokes T, Sandhu E, Pepper RJ, et al. Induction treatment of ANCAassociated vasculitis with a single dose of rituximab. Rheumatology (Oxford). 2014;53(8):1395-1403.

52. Evens AM, Jovanovic BD, Su YC, et al. Rituximab-associated hepatitis B virus (HBV) reactivation in lymphoproliferative diseases: metaanalysis and examination of FDA safety reports. Ann Oncol. 2011;22(5): $1170-1180$.

53. Westhoff TH, Jochimsen F, Schmittel A, et al. Fatal hepatitis B virus reactivation by an escape mutant following rituximab therapy. Blood. 2003;102(5):1930.

54. Sarrecchia C, Cappelli A, Aiello P. HBV reactivation with fatal fulminating hepatitis during rituximab treatment in a subject negative for $\mathrm{HBsAg}$ and positive for $\mathrm{HBsAb}$ and $\mathrm{HBcAb}$. J Infect Chemother. 2005; 11(4):189-191.

55. Perceau G, Diris N, Estines O, Derancourt C, Levy S, Bernard P. Late lethal hepatitis B virus reactivation after rituximab treatment of low-grade cutaneous B-cell lymphoma. Br J Dermatol. 2006;155(5): 1053-1056.

56. Cooper N, Arnold DM. The effect of rituximab on humoral and cell mediated immunity and infection in the treatment of autoimmune diseases. Br J Haematol. 2010;149(1):3-13.

57. Huang YH, Hsiao LT, Hong YC, et al. Randomized controlled trial of entecavir prophylaxis for rituximab-associated hepatitis B virus reactivation in patients with lymphoma and resolved hepatitis B. J Clin Oncol. 2013;31(22):2765-2772.

58. Huang H, Li X, Zhu J, et al. Entecavir vs lamivudine for prevention of hepatitis $\mathrm{B}$ virus reactivation among patients with untreated diffuse large B-cell lymphoma receiving R-CHOP chemotherapy: a randomized clinical trial. JAMA. 2014;312(23):2521-2530.

59. Casulo C, Maragulia J, Zelenetz AD. Incidence of hypogammaglobulinemia in patients receiving rituximab and the use of intravenous immunoglobulin for recurrent infections. Clin Lymphoma Myeloma Leuk. 2013;13(2):106-111.

60. Besada E. Routine pneumocystis pneumonia prophylaxis in patients treated with rituximab? Chest. 2013;144(1):359-360.

61. Martin-Garrido I, Carmona EM, Specks U, Limper AH. Pneumocystis pneumonia in patients treated with rituximab. Chest. 2013;144(1): $258-265$.

62. Green H, Paul M, Vidal L, Leibovici L. Prophylaxis of pneumocystis pneumonia in immunocompromised non-HIV-infected patients: systematic review and meta-analysis of randomized controlled trials Mayo Clin Proc. 2007;82(9):1052-1059.
ImmunoTargets and Therapy

\section{Publish your work in this journal}

ImmunoTargets and Therapy is an international, peer-reviewed open access journal focusing on the immunological basis of diseases, potential targets for immune based therapy and treatment protocols employed to improve patient management. Basic immunology and physiology of the immune system in health, and disease will be also covered. In addition, the journal will focus on the impact of manage-

\section{Dovepress}

ment programs and new therapeutic agents and protocols on patient perspectives such as quality of life, adherence and satisfaction. The manuscript management system is completely online and includes a very quick and fair peer-review system, which is all easy to use. Visit http://www.dovepress.com/testimonials.php to read real quotes from published authors. 